\title{
Swelling of a Polymer Brush by a Poor Solvent
}

\author{
P. D. GAllaGHer, ${ }^{1}$ S. K. SATIJA, ${ }^{1}$ A. KARIM, $^{2}$ J. F. DOUGLAS, ${ }^{2}$ L. J. FETTERS $^{3}$ \\ ${ }^{1}$ National Institute of Standards and Technology (NIST) Center for Neutron Research, Gaithersburg, Maryland 20899 \\ ${ }^{2}$ Polymers Division, NIST, Gaithersburg, Maryland 20899 \\ ${ }^{3}$ Cornell University, Department of Chemical and Biomolecular Engineering, Ithaca, New York 14853
}

Received 29 June 2004; accepted 21 July 2004

DOI: 10.1002/polb.20257

Published online in Wiley InterScience (www.interscience.wiley.com).

\begin{abstract}
The addition of a small amount of a poor solvent impurity (methanol) to a theta solvent (cyclohexane) is found to cause appreciable swelling $(\approx 30 \%$ increase of the average brush height) in a model end-grafted polystyrene (PS) brush layer. This unusual type of swelling is not observed if octadecyltrichlorosilane (OTS) is first grafted to the portion of the silicon substrate uncovered by the grafting end-groups of the PS chains. Brush swelling in the absence of OTS surface protection is interpreted as arising from a segregation of methanol to the solid substrate and the resulting modification of the polymer-surface interaction. We also observe that the addition of a small amount of methanol to an adsorbed PS layer exposed to cyclohexane causes rapid film delamination from the silicon substrate. Together these observations imply a strong influence of surface active impurities on the structure and adhesive stability of polymer layers. () 2004 Wiley Periodicals, Inc.* J Polym Sci Part B: Polym Phys 42: 4126-4131, 2004
\end{abstract}

\section{INTRODUCTION}

The stability and structure of polymer films on solid substrates is often found to be sensitive to environmental conditions. For example, a small amount of humidity can cause a polymer film to delaminate as moisture from the air diffuses through the film to the substrate. ${ }^{1}$ Displacement of the polymer film can occur rapidly when it is exposed to a solvent containing a component with a high affinity for the solid substrate. Indeed, polymer films are often floated off their substrates for further examination based on this phenomenon. We also anticipate that this displacement effect is important in chain layers end-grafted to solid substrates (brushes) where displacement

Correspondence to: P. D. Gallagher (E-mail: patrick. Gallagher@nist.gov)

Journal of Polymer Science: Part B: Polymer Physics, Vol. 42, 4126-4131 (2004) (C) 2004 Wiley Periodicals, Inc. *This article is a US Government work and, as such, is in the public domain in the United States of America. (i.e., swelling) occurs locally on the scale of the polymer chain because of the constraint of chain grafting. (We use the term brush swelling to refer to any thermodynamic effect (e.g., solvent quality, polymer-surface interaction) that causes the brush layer to expand in dimensions.) If the surface segregating solvent (impurity) has a repulsive interaction with the polymer (i.e., the polymer is immiscible with the impurity solvent), then this surface segregation should lead to a swelling of the grafted chain layer, a mechanism similar to the stretching of ordering block copolymer lamellar microphases arising from a corresponding repulsive interblock interaction. ${ }^{2}$ Notably, grafted or strongly adsorbed chain layers are involved in many applications (chromatography, colloid stabilization, modification of surface wetting characteristics, and other surface properties such as friction and adhesion, surface modification of filler particles in composites, emulsion stabilization, etc.) so that swelling induced by a small amount of impurity has basic scientific and 
engineering significance (see ref. 3 for a more complete listing of polymer brush applications).

In this article, we consider model deuterated polystyrene grafted chain layers swollen by the theta solvent cyclohexane $(\mathrm{CH})$ and we add small amounts of the poor solvent methanol (Me) that has a strong affinity for the silica substrate on which the chains are grafted. This type of grafted layer has been carefully characterized in previous work in the absence of the Me impurity ${ }^{4}$ and should provide a good model system for quantitatively investigating the modification of the structure of grafted polymer layers because of impurity enrichment near the solid substrate. Octadecyltrichlorosilane (OTS) was also grafted onto the silicon substrate after grafting the polymer layer to block Me enrichment of the substrate surface. We report observations on the swelling of polystyrene (PS) layers in $\mathrm{CH}$ and $\mathrm{CH}-\mathrm{Me}$ mixed solvents both with and without the OTS surface treatment. The solvent displacement effect is also demonstrated as a qualitative effect in polymer films subjected to environmental exposure to a small concentration of a surface active impurity. Specifically, we consider some simple film-washing experiments performed at room temperature on spun-cast PS films adsorbed on the same silicon wafers used in our grafted chain layer measurements. Little change in the PS films was observed upon exposure to a pure cyclohexane solution, whereas a small concentration of Me added to the wash solution caused the film to rapidly delaminate from the substrate.

\section{EXPERIMENTAL}

Deuterated polystyrene (dPS) with a trichlorosilane end-group [dPS- $\mathrm{SiCl}_{3} ;$ molecular mass, (weight-average molecular weight) $M_{\mathrm{w}}=85,000$; polydispersity, $M_{\mathrm{w}} /$ (number-average molecular weight) $M_{\mathrm{n}}<1.05$ ] was end-grafted onto a single crystal silicon surface. The silicon wafers were acid-cleaned in a $70 \% \mathrm{H}_{2} \mathrm{SO}_{4} / 30 \% \mathrm{H}_{2} \mathrm{O}_{2}$ (w/w) acid mixture, as described previously. After grafting, the films were kept in toluene for several days to dissolve away any nongrafted polymer. This procedure ensures that essentially all of the chains in the polymer layer are chemically end grafted to the surface and that no polymer loss occurs in our mixed-solvent brush swelling measurements.

$\mathrm{X}$-ray reflectivity measurements on the dried polymer film indicated a smooth film having a film thickness of $h=12 \mathrm{~nm}$. This corresponds to a polymer surface coverage $\Gamma=10 \mathrm{mg} / \mathrm{m}^{2}$ or a dimensionless surface grafting density $\sigma=(b / d)^{2}$ $=0.035$, where $b=0.67 \mathrm{~nm}$ is the statistical segment size and $d$ is the average distance between grafting sites. This grafting density is relatively high and it is conventional to refer to such layers as brushes. ${ }^{4-6}$ In the polymer film displacement measurements, the PS was adsorbed from a $1 \%$ polystyrene solution (by mass) onto an acid cleaned $\mathrm{Si}$ wafer and the film was then washed with pure cyclohexane, leaving a residual persistently adsorbed PS layer. The wafers were then divided into pieces and exposed to pure $\mathrm{CH}$ and a mixture of $\mathrm{CH}$ and $\mathrm{Me}$, as in the grafted polymer layer measurements.

The formation of a relatively high grafting density brush through grafting from solution requires a moderate polymer-surface attractive interaction so that the chains are neither depleted from the surface nor strongly adsorbed and it is also necessary for the solvent quality to be poor (below the theta temperature where the second osmotic pressure virial coefficient vanishes, $T_{\theta}$ ) to diminish repulsive interchain interactions that reduce the saturation grafting density. ${ }^{7}$ Our acidcleaning of the Si substrates and the subsequent chain grafting procedure follows the method described in ref. 7 and, thus, the PS chains in our brush layer should have a moderate attraction for the Si substrate in the absence of OTS functionalization or Me enrichment.

Neutron reflectometry (NR) measurements were performed on the NG7 instrument at the National Institute of Standards and Technology (NIST). The sample cell consists of the Si block with the grafted dPS film placed opposite a quartz glass block separated by a spacer with the solvent between the blocks. Measurements were made with the incident beam through one edge of the single crystal, reflected from the Si-brush-solvent interface, and back through the Si surface to the detector. The neutron wavelength $\lambda$ was fixed at $0.475 \mathrm{~nm}$ and the angle of incidence $\theta$ was varied while measuring the reflected neutron intensity as a function of wavevector magnitude, $Q=(4 \pi / \lambda)$ $\sin \theta$. The incident beam collimation was also varied to maintain a constant beam footprint on the sample. The scattering cell temperature $(T)$ was controlled to within $0.02{ }^{\circ} \mathrm{C}$ by enclosing it in a thermostated water bath.

We remind the reader that $\mathrm{CH}$ is a theta-solvent for $\mathrm{dPS}\left(T_{\theta} \approx 35^{\circ} \mathrm{C}\right)$, whereas $\mathrm{Me}$ is a poor or nonsolvent. In the case of a small concentration of methanol, we expect $\mathrm{CH}$ to preferentially enrich the dPS chains in solution so that the Me can be 
expected to have little influence on the chain dimensions. Small angle neutron scattering (SANS) measurements of the chain dimensions were also performed at a temperature well above the $T_{\theta}$ of $\mathrm{PS}$ in $\mathrm{CH}\left(T=55^{\circ} \mathrm{C}\right)$ for a range of $\mathrm{Me}$ concentrations (volume fraction), $\phi_{\mathrm{Me}} \leq 0.05$. No detectable change in the chain radius of gyration $\left(R_{\mathrm{g}}\right)$ was observed in these measurements, consistent with local $\mathrm{CH}$ enrichment and methanol depletion near the polymer chains. $\mathrm{CH}-\mathrm{Me}$ mixtures are partially miscible, and can phase separate below an upper critical point temperature of approximately $45{ }^{\circ} \mathrm{C}$. Brush measurements discussed in this article were performed at $T=55{ }^{\circ} \mathrm{C}$ and at concentrations far from the critical composition so that the solvent mixture is fully miscible at all concentrations studied here. We did not expect the bulk phase behavior of the mixture to be affected by the surface interactions, and we verified visually that phase separation of the liquid mixture occurred near $45^{\circ} \mathrm{C}$ at compositions near the critical composition.

\section{RESULTS AND DISCUSSION}

In Figure 1(a) we show normalized NR data for a PS grafted layer on a Si substrate over a range of methanol concentrations, $\phi_{\mathrm{Me}}=0.0-0.2$. The first minimum of the reflectivity curves decreases to a smaller $Q$ with an increasing $\phi_{\mathrm{Me}}$, indicating that the polymer brush becomes progressively swollen with an increasing concentration of the poor solvent impurity Me in cyclohexane.

The density profile of a similar dPS grafted layer in pure $\mathrm{CH}$ was studied previously ${ }^{4}$ and the general features of these films are expected to be qualitatively similar in form, apart from the apparent change in film thickness. Because the determination of $h$ from the reflectivity minimum is insensitive to model assumptions made about the form of the segmental density profile, ${ }^{8}$ we focus in this initial exploratory article on the relative change of the thickness $h$ of the brush layer arising from the Me impurity. Thus, the ratio of the first minimum $\left(Q_{\min }\right)$ position in the reflectivity curve for the brush layer exposed to pure $\mathrm{CH}$ to that for the $\mathrm{CH} / \mathrm{Me}$ mixture exposed to the same brush layer determines the relative change in the brush height $h$ to the brush height in pure $\mathrm{CH}\left(h_{\theta}\right)$ as a function of $\phi_{\mathrm{Me}}$. The uncertainty of this $h$ estimate is limited by the instrumental $Q$ resolution in determining the reflectivity minimum (typically $0.01 \mathrm{~nm}^{-1}$ ). Our findings for $h / h_{\theta}$ are shown in Figure 2 for this brush and for a similar

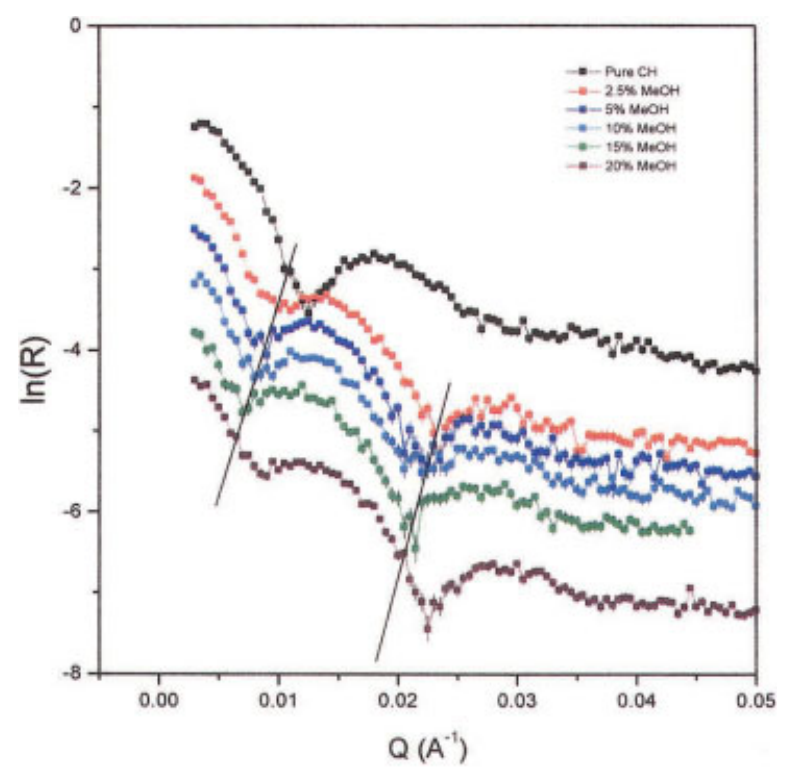

(a)

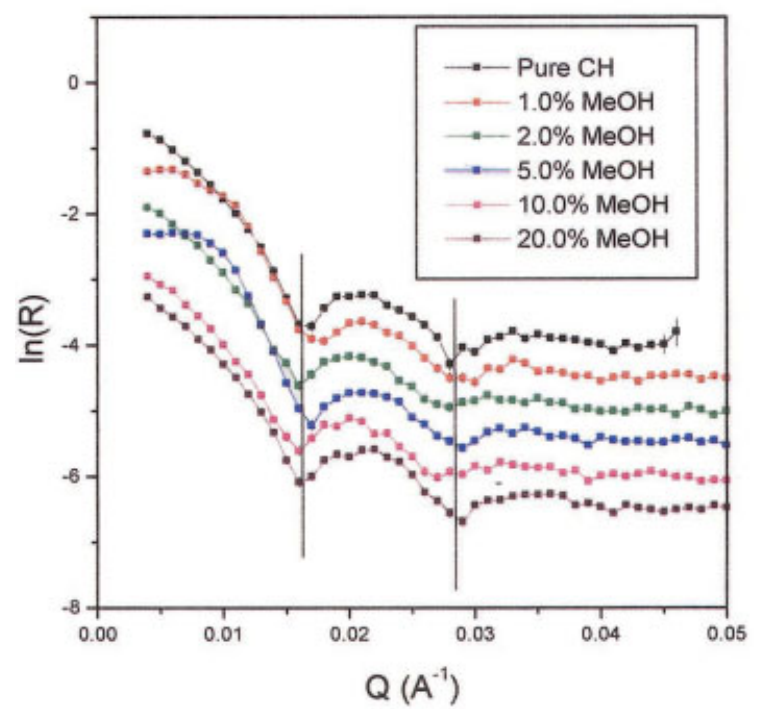

(b)

Figure 1. Normalized neutron reflectivity data for a grafted polystyrene chain layer swollen by a mixed solvent of cyclohexane and methanol. Data points in each set are connected by a line for clarity. The methanol concentration of the mixture is indicated in each figure, and data from different concentrations are offset vertically for clarity; (a) and b) correspond to chains grafted on Si wafers without and with OTS treatment, respectively. Silicon surface modification by grafting OTS follows the grafting of dPS to the substrate. OTS treatment renders the portion of the Si surface that is uncovered by polymer more hydrophobic so that the Me affinity for the solid substrate is diminished. Slanted lines are drawn to show the general trend in the change in the position of the first reflectivity minimum $Q_{\min }$. Values to determine changes in PS brush height are computed from the minima of actual data sets with standard reflectivity principles (see refs. 4 and 8 for details). 


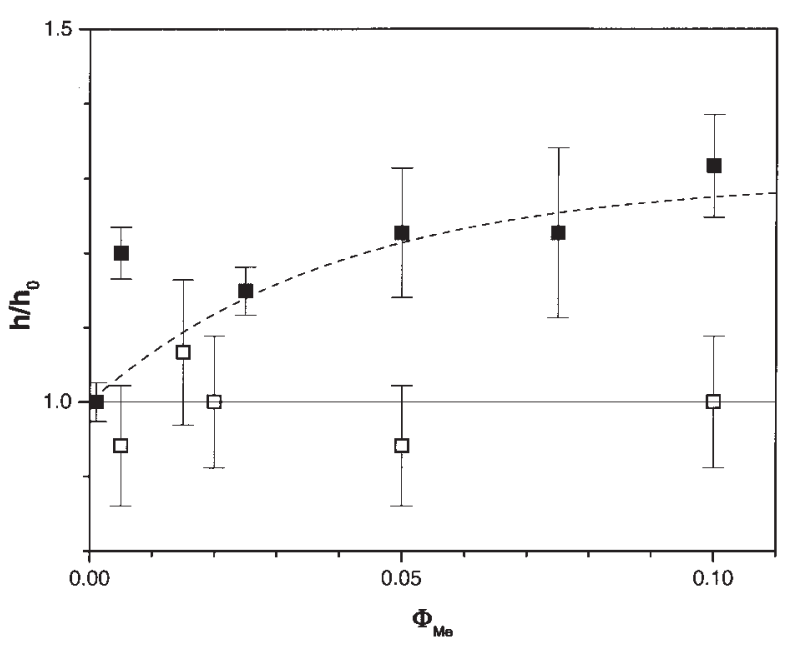

(a)

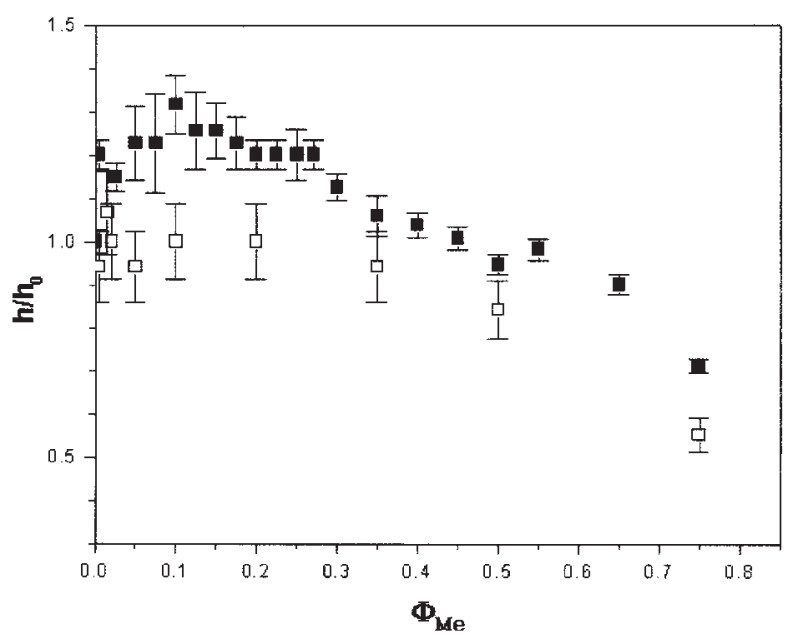

(b)

Figure 2. Mean thickness, $h$, of a polystyrene chain layer grafted on a silicon wafer and swollen by cyclohexane mixed with a varying amount of methanol impurity. The swelling is described relative to the thickness, $h_{o}$, of the same film swollen in pure $\mathrm{CH}:(-)$ denotes measurements without OTS, whereas $(\square)$ denotes the OTS treated grafted layers. Trend lines are shown in (a) to guide the eye; (b) the same data over an extended concentration range of added methanol. Note the general trend of chain contraction for methanol concentrations above $20 \%$, corresponding to the saturation of the methanol surface enrichment and the reduction of solvent quality because of the segregation of the Me to the polymer chains. The high Me concentration regime was studied in a previous article. ${ }^{12}$

brush with OTS grafted onto the surface after the PS chains to protect the surface from Me segregation (see discussion below). We observe that $h / h_{\theta}$ increases sharply with the addition of $\mathrm{Me}$ and that this increase continues up to $\phi_{\mathrm{Me}} \approx 0.2$. At higher $\phi_{\mathrm{Me}}$, we observe that $h$ decreases, as we would expect for a polymer exposed to an increasingly poor solvent and for saturation of the $\mathrm{Si}$ surface by Me. The Me-induced brush swelling is thus limited to a concentration range, $\phi_{\mathrm{Me}}<0.2$, and we limit the following discussion to this region.

Renormalization group calculations ${ }^{9}$ of the swelling of grafted chain layers in the limit of low grafting density indicate a substantial dependence of the composition profile of the grafted layer on the polymer-surface interaction, whereas little dependence on the polymer-surface interaction is expected in the limit of high grafting density brushes. Because the grafting density of real grafted layers usually lies between these extreme models, ${ }^{3}$ we expect that our semidilute concentration brush layers should involve some dependence of the layer thickness on the polymer-surface interaction. In the limit of low grafting density, where the effect should be largest, the change of grafted layer thickness is predicted to be as large as 50\% for a change from an attractive, but nonadsorbing surface (adsorption theta point ${ }^{9}$ ), to a repulsive surface and this limiting result should provide a rough upper bound on the extent of swelling in (nonadsorbed) grafted polymer layers arising from a variable polymersurface interaction. ${ }^{9}$ Both simulation ${ }^{10}$ and experiment ${ }^{3}$ indicate that the influence of the polymer-surface interaction on the brush profile shape is especially large in theta solvents (much higher grafting densities are necessary for chain stretching to occur in theta solvents because of the diminished interchain interactions ${ }^{7}$ ) so that we expect an appreciable effect on brush swelling from Me segregation to the substrate for our theta solvent swollen brush measurements. As a control measurement on our interpretation of swelling arising from a Me-induced modification of the polymer-surface interaction, we followed the grafting of the dPS chains to the Si substrate by grafting OTS onto the ungrafted portion of the $\mathrm{Si}$ substrate. We then performed our NR measurements on these grafted layers in mixed $\mathrm{CH}-\mathrm{Me}$ solvents as before under otherwise identical conditions.

The reflectivity data for the brush with the OTS modified surface is shown in Figure 1(b). Strikingly, there is essentially no change in the thickness of the grafted layer for this range of $\phi_{\mathrm{Me}}$, as in the solution PS measurements of $R_{\mathrm{g}}$. The relative thickness of the dPS grafted layers with the OTS modified surface as a function of $\phi_{\mathrm{Me}}$ is shown in Figure 2. For $\phi_{\mathrm{Me}}>0.2$ the grafted layer begins to contract steadily with the 
progressive intrusion of Me into the grafted chain layer.

We attempted to directly observe the tendency of Me to segregate to the Si substrate through a selective deuteration of the solvent. Unfortunately, the deuteration of Me leads to a change in solvent quality for $\mathrm{dPS}^{11}$ so we restrict our discussion to the deuteration of the $\mathrm{CH}$. NR measurements presented earlier show that the deuteration of the $\mathrm{CH}$ has little effect on $h$ and thus on the solvent quality of the dPS in deuterated $\mathrm{CH}^{4,12}$ These measurements also show that the $\mathrm{CH}$ enriched the grafted chain layer even for rather large Me concentrations $\left(\phi_{\mathrm{Me}}=1 / 2\right) .{ }^{12} \mathrm{Al}$ though it is difficult to resolve a very thin layer of enrichment of Me near the Si substrate, these measurements do safely rule out the existence of a large enrichment layer of $\mathrm{Me}(h>0.5 \mathrm{~nm})$. Apparently, only a small amount of Me adsorbed near the silica substrate is responsible for the substantial swelling of the grafted layer shown in Figure 1(a).

Our observations suggest the following explanation for the impurity induced swelling of the grafted chain layer. Although $\mathrm{CH}$ is a much better solvent for dPS than Me at $T=55{ }^{\circ} \mathrm{C}$ and thus selectively segregates to the dPS grafted layer, a thin layer of $\mathrm{Me}$ can segregate beneath the main body of the brush to the native oxide layer on the silicon substrate (this type of segregation was suggested previously by Johner and Marques ${ }^{13}$ ). Despite the limited extent of the Me layer, it can exert a strong influence on the structure of the grafted layer through a modification of the polymer-surface interaction. Me is a poor solvent for dPS and its presence at the Si surface should change the attractive dPS-Si surface interaction (necessary for the initial formation of the dPS layer) into a relatively repulsive interaction, leading to an expansion of the grafted chain layer under essentially constant solvent quality conditions. The grafting of OTS to the available silicon oxide surface underneath the brush can block the Me segregation and thus inhibit the film swelling from the surface interaction modification. These observations clearly establish the importance of polymer-surface interactions on the dimensions of grafted chain layers, as indicated by renormalization group calculations of grafted chains in the limit of low grafting densities or weak excluded volume interactions. ${ }^{9}$ The maximum observed surface-interaction induced swelling of the brush layer $(<30 \%)$ is somewhat less than the predictions of the dilute solution theory $(50 \%)$, consistent with an intermediate grafting density and a relatively weak excluded volume interaction in the theta-solvent swollen brush. We contrast the findings of this article with previous observations of brush swelling in mixed solvents, where large changes in brush dimensions have been observed in conjunction with preferential segregation by the solvent components to the polymer chains,${ }^{12,14}$ in qualitative similarity to bulk polymer solutions. In these other studies, the variation of the effective polymer-polymer interactions with solvent composition drives the brush swelling rather than the polymer-surface interaction.

As a final illustration of the effect of a selective segregation of a surface active impurity to a solid substrate, we consider the qualitative influence of small concentrations of $\mathrm{Me}$ on the stability of (ungrafted) PS films. The PS films were formed by adsorbing regular polystyrene (without the $\mathrm{SiCl}_{3}$ end group) polymer from a dilute $\mathrm{CH}$ solution (polymer concentration was $1 \%$ by mass) onto an acid-cleaned Si wafer. The macroscopically thick films were then washed in pure $\mathrm{CH}$ at room temperature to leave a residual thin film of a persistently adsorbed polymer, as measured by a water contact angle of $90 \pm 5^{\circ}$. The film thickness of the layer was not measured, but this property is less relevant here. It is worth noting that at higher temperatures the $\mathrm{CH}$ becomes a better solvent and one would expect the film to eventually wash off. However, from extensive experience with solvent washing of polymer films we know that this process is very slow at temperatures near $55{ }^{\circ} \mathrm{C}$, so that a residual film remains on the substrate. These observations indicate an appreciable attractive polymer-surface interaction. The wafer with the thin PS layer was then broken into pieces that were exposed to a solvent mixture (also at room temperature) of $\mathrm{CH}$ and a very small fraction of $\mathrm{Me}\left(\phi_{\mathrm{Me}}=0.01\right.$ and 0.001 ) for approximately $1 \mathrm{~min}$. (Notably, Me is still completely miscible in $\mathrm{CH}$ at room temperature at these low concentrations.) In contrast to the case of pure $\mathrm{CH}$, where the PS layer remained persistently attached for $\phi_{\mathrm{Me}}=0.01$ and 0.001 , the PS film apparently washed off immediately, leaving a bare silica wafer as measured by a near zero water contact angle $\left(<5^{\circ}\right)$. Thus, the presence of small amounts of Me in the mixture, presumably through the segregation of Me to the solid substrate, has a dramatic effect on the film stability, and a macroscopic rather than a microscopic displacement of the film is achieved through a modification of the effective polymer-surface interaction caused by 
the Me. This effect provides further evidence for the significance of impurity segregation on the structure and stability of thin polymer films.

\section{CONCLUSIONS}

In conclusion, we find that a very small amount of surface active impurity can have a large impact on the dimensions of polymer brush layers and the adhesion stability of polymer layers. This brush swelling effect is unexpected from the perspective of ideal brush layers, where little sensitivity to polymer-surface interaction has been presumed to exist. Apparently, real grafted layers with physically accessible grafting densities can be stimulated to expand or contract through the addition of surface active molecules. By extension, we should be able to observe brush contraction upon the addition of a small amount of a surface active impurity that is a good solvent for the polymer. These additive effects potentially provide a means to actuate changes in the brush structure in applications. Our qualitative finding that a minute amount of surface active impurity can cause the delamination of polymer layers and an associated loss of adhesion also has many technological implications.

\section{REFERENCES AND NOTES}

1. Kinloch, J. Adhesion and Adhesives: Science and Technology; Chapman and Hall: New York, 1987); see also: Kent, M. S.; McNamara, W. F.; Fein, D.; Domeier, L. A.; Wong, P. Y. J Adhes 1999, 69, 121-138; Wu, W.; Orts, W. J.; Majkrzak, C. J.; Hunston, D. L. Polym Eng Sci 1995, 35, 1000; Becktan, N. C.; Wu, W.; Wallace, W. E.; Davis, G. T. J Polym Sci Part B: Polym Phys 1998, 36, 155.

2. Almdal, K.; Rosedale, J.; Bates, F. S.; Wignall, G. D.; Fredrickson, G. H. Phys Rev Lett 1990, 65, 1112; Papadakis, C. M.; Almdal, K.; Motensen, K.; Posselt, D. Europhys Lett 1986, 36, 289; Ohta, T.; Kawasaki, K. Macromolecules 1986, 19, 2621.

3. Kent, M. S. Macromol Rapid Comm 2000, 21, 243.

4. Karim, A.; Satija, S. K.; Douglas, J. F.; Ankner, J. F.; Fetters, L. J. Phys Rev Lett 1994, 73, 3407; see also: Karim, A.; Douglas, J. F.; Horkay, F.; Fetters, L. J.; Satija, S. K. Phys B: Condens Matter 1996, 221, 331; Douglas, J. F.; Karim, A.; Kent,
M. S.; Satija, S. K. "Polymer Brushes: Structure and Dynamics"; In Encyclopedia of Materials: Science and Technology; Elsevier, New York, 2001; pp 7218-7223.

5. Halperin, A.; Tirrel, M.; Lodge, T. P. Adv Polym Sci 1992, 100, 33.

6. deGennes, P. G. Macromolecules 1980, 13, 1069; Milner, S. T. Science 1991, 251, 905; Milner, S. T.; Witten, T. A.; Cates, M. E. Macromolecules 1988, 21, 2610; Milner, S. T.; Wang, Z. -G.; Witten, T. A. Macromolecules 1989, 22, 489; Zhulina, E. B.; Borisov, O. V.; Pryamitsyn, V. A.; Birshstein, T. M. Macromolecules 1991, 24, 140. The brush theory is restricted to moderate concentrations that are sufficiently high for mean field theory to apply, but not so high that the finite extensibility chains invalidates the model. See: Shim, D. F. K.; Cates, M. E. J. Physique 1989, 50, 3535.

7. Karim, A.; Tsukruk, V. V.; Douglas, J. F.; Satija, S. K.; Fetters, L. J.; Reneker, D. H.; Foster, M. D. J. Physique II 1995, 5, 1441; Douglas, J. F.; Lipman, R.; Karim, A.; Granick, S. Polym Mat Sci Engr 1997, 77, 644. Nonequilibrium effects on polymer conformations associated with strong adsorption are described by Douglas, J. F. et al., J Phys: Condens Matter 1997, 9, 7699. These nonequilibrium effects are expected to show nonuniformity in chain grafting because of the limited accessibility of the substrate by the grafting chain ends. This effect has not been checked experimentally, however.

8. Russell, T. P. Mater Sci Reports 1990, 5, 171.

9. Adamuti-Trache, M.; McMullen, W. E.; Douglas, J. F. J Chem Phys 1996, 105, 4798. There has been some limited SCF modeling on polymer-surface interaction effects on grated layer swelling: Cosgrove, T.; Heath, T.; van Lent, B.; Leermakers, F.; Scheutjens, J. Macromolecules 1987, 20, 1692.

10. Binder, K.; Lai, P.; Wittmer, J. Faraday Discuss 1994, 98, 97; Lai, P.; Binder, K. J Chem Phys 1992, 97, 586.

11. Houessou, C.; Guenoun, P.; Gastaud, R.; Perrot, F.; Beysens, D. Phys Rev A: At Mol Opt Phys 1985, 32, 1818.

12. Satija, S. K.; Gallagher, P.; Karim, A.; Fetters, L. J. Phys B: Condens Matter 1998, 248, 204. This work considers the case of PS brushes in methanol and cyclohexane in the limit of high methanol concentration where the surface methanol concentration is saturated and where solvent segregation to the polymer predominates the brush swelling.

13. Johner, A.; Marques, C. M. Phys Rev Lett 1992, 69, 1827.

14. Auroy, P.; Auvray, L. Macromolecules 1992, 25, 4134. 